\title{
Loss of Control Eating During Pregnancy is Associated With Excessive Gestational Weight Gain Among Individuals With Overweight and Obesity
}

Michele Levine ( $\sim$ levinem@upmc.edu )

University of Pittsburgh https://orcid.org/0000-0002-1054-3856

Rebecca L. Emery

University of Pittsburgh

Rachel Conlon

University of Pittsburgh School of Medicine

Jennifer Grace

University of Pittsburgh Medical Center

Gina M. Sweeny

University of Pittsburgh Medical Center

Bang Wang

University of Pittsburgh

Yu Cheng

University of Pittsburgh

\section{Research Article}

Keywords: pregnant, gestational weight gain, loss of control eating, binge eating

Posted Date: February 21st, 2022

DOI: https://doi.org/10.21203/rs.3.rs-1155506/v1

License: (c) (i) This work is licensed under a Creative Commons Attribution 4.0 International License. Read Full License 


\section{Abstract}

Purpose: Excessive gestational weight gain (GWG) predicts negative health outcomes among individuals with overweight or obesity. Loss of control eating (LOC), the ingestion of food associated with being unable to control eating, is the core psychopathology of binge eating disorders. We evaluated the contribution of LOC to GWG among pregnant individuals with prepregnancy overweight/obesity.

Methods: In a prospective longitudinal study, individuals with prepregnancy $B M I \geq 25(N=257)$ were interviewed monthly to assess LOC and reported demographic, parity and smoking information. GWG was abstracted from medical records.

Results: Among individuals with prepregnancy overweight/obesity, 39\% endorsed LOC prior to or during pregnancy. After adjusting for factors that have previously been linked to GWG, LOC during pregnancy, uniquely predicted higher GWG and greater likelihood of exceeding GWG recommendations. Participants with prenatal LOC gained $3.14 \mathrm{~kg}(p=0.03)$ more than did those without LOC during pregnancy and 78.7\% $(n=48 / 61)$ exceeded IOM guidelines for GWG. The frequency of LOC episodes was also associated with greater weight gain.

Conclusions: Prenatal LOC is common among pregnant individuals with overweight/obesity and predicts greater GWG and increased likelihood of exceeding IOM GWG guidelines. LOC may represent a modifiable behavioral mechanism to prevent excessive GWG among individuals at risk for adverse pregnancy outcomes.

\section{Introduction}

Excessive gestational weight gain (GWG) robustly predicts obstetric complications (Rosenberg, Garbers, Chavkin, \& Chiasson, 2003), greater postpartum weight retention (Linné, Dye, Barkeling, \& Rössner, 2004), and adverse neonatal outcomes (Chen \& Chauhan, 2019). Thus, preventing excessive gestational weight gain (GWG) can improve the health of mothers and children. However, more than 45\% (Goldstein et al., 2017; Schieve, Cogswell, \& Scanlon, 1998) of pregnant people exceed guidelines established by the Institute of Medicine (IOM) for GWG (Institute of Medicine \& Committee to Reexamine Pregnancy Weight Guidelines, 2009). Compared to those who begin pregnancy with normal weight, those who begin pregnancy with overweight or obesity are more likely to gain excessive gestational weight during pregnancy (Linné et al., 2004) and retain a larger amount of gestational weight postpartum (Amorim, Rössner, Neovius, Lourenço, \& Linné, 2007; Vesco et al., 2009). To optimize interventions that address excess weight gain, it is critical to identify behaviors related to GWG, particularly among individuals who begin pregnancy with overweight or obesity.

One modifiable mechanism that may contribute to excessive GWG is loss of control eating (LOC). LOC refers to the ingestion of food with an associated experience of being unable to control one's eating and is the core psychopathology of bulimia nervosa and binge eating disorders (Latner, Hildebrandt, Rosewall, Chisholm, \& Hayashi, 2007). LOC is more common among women than men, affecting up to $30 \%$ of women between the ages of 18 and 35 (Striegel-Moore et al., 2009). LOC is associated with increased daily calorie intake, frequent overeating episodes (Engel et al., 2009; Yanovski et al., 1992) and higher levels of depressive symptoms (Colles, Dixon, \& O'Brien, 2008), which themselves are associated with weight gain and obesity (Marcus \& Wildes, 2009). Importantly, LOC is the most frequently reported disordered eating behavior during pregnancy (Bulik et al., 2007; Morgan, Lacey, \& Chung, 2006; Soares et al., 2009). Although other serious disordered eating behaviors often improve during pregnancy (Blais et al., 2000; Bulik et al., 2007; Crow, Agras, Crosby, Halmi, \& Mitchell, 2008), LOC does not improve during pregnancy (Bulik et al., 2007). To date, rates of LOC during pregnancy range from 8.4 to $36 \%$, depending on the method of assessment and population studied (Easter et al., 2013; Hecht, Schwartz, Miller-Matero, Braciszewski, \& Haedt-Matt, 2020; Kolko, Emery, Marcus, \& Levine, 2017; Micali, Al Essimii, Field, \& Treasure, 2018). For example, we previously reported that $24 \%$ of pregnant people with overweight or obesity endorsed LOC during pregnancy (Kolko et al., 2017). 
In addition to evidence that LOC persists during pregnancy, converging evidence suggests that LOC and binge eating may be associated with excessive GWG (Park et al., 2015) although the link to excessive GWG has not been consistently noted (Hecht et al., 2020). Initial evidence suggested that people who binge eat during pregnancy report more worry about GWG (Nunes, Pinheiro, Camey, \& Schmidt, 2012; Swann et al., 2009), and worry, in turn, has been associated with higher GWG (Heery, Wall, Kelleher, \& McAuliffe, 2016). Recently, Micali and colleagues (Micali et al., 2018) found that those who experienced LOC in pregnancy gained an average of $3.74 \mathrm{~kg}$ more than those who denied experiencing LOC in pregnancy. Although these findings support a possible link between LOC in pregnancy and elevated risk for higher GWG, previous studies have been limited by the retrospective reports eating behaviors assessed at the end of pregnancy rather than across pregnancy (Hecht et al., 2020; Micali et al., 2018), the use of self-reported LOC (Hecht et al., 2020; Heery et al., 2016; Micali et al., 2018; Nunes et al., 2012; Park et al., 2015) and the assessment of LOC at only one point during pregnancy (Hecht et al., 2020; Micali et al., 2018), limiting the ability to fully characterize the impact of LOC and changes in LOC across the full gestational period on GWG. Moreover, previous studies have been conducted among samples of pregnant people enrolled in interventions designed to promote weight gains within the IOM guidelines (Tang, Andres, West, Lou, \& Krukowski, 2020) or using convenience samples of pregnant people who had not been selected based on risk of aberrant eating or for excessive GWG. The impact of LOC in the context of individuals at highest risk of negative obstetric outcomes and the ways in which LOC may change across pregnancy remain unclear.

Given that LOC is associated with difficulty managing weight over time (Gorin et al., 2008) and tends to persist during pregnancy, we reasoned LOC may be a critical and modifiable component of GWG. Thus, the present study sought to determine the contribution of LOC, frequently assessed across pregnancy and measured using a standard clinical interview adapted for the perinatal period (Emery, Grace, Kolko, \& Levine, 2017) to GWG among people who began pregnancy with overweight or obesity. We hypothesized that LOC during pregnancy would be associated with larger total GWG and with an increased likelihood of exceeding IOM guidelines for GWG. We also predicted that the frequency of LOC episodes during pregnancy would be associated with an increased likelihood of exceeding IOM guidelines for GWG and with larger total GWG.

\section{Materials And Methods}

\section{Participants and procedures}

Pregnant individuals $(N=257)$ were recruited from obstetric clinics associated with a large urban hospital. Individuals were eligible if they had a pre-pregnancy body mass index (BMI) $\geq 25 \mathrm{~kg} / \mathrm{m}^{2}$, were $12-20$ weeks gestation, were $\geq 14$ years old, and had a singleton pregnancy. Exclusion criteria were use of weight-affecting medications (e.g., steroids) or medical conditions (e.g., thyroid disorders), participation in weight-management programming, type 1 diabetes, or psychiatric symptoms requiring immediate treatment.

As shown in Figure 1, between September 2012 and January 2017, a total of 1023 individuals were contacted about the study and 995 were screened for eligibility. Those determined to be eligible came for an in-person baseline assessment (T0), during which they completed questionnaires, height and weight measurements, and interviews. Participants then completed 5 monthly telephone interview assessments of LOC (T1-T5). As shown in Figure 1, 253 (98.4\% of eligible), 251 (98.8\% of eligible), 245 (98.0\% of eligible), 239 (96.8\% of eligible), and 202 ( $96.2 \%$ of eligible) participants completed monthly interviews as scheduled. A total of 198 participants completed 6 monthly interview assessments of LOC and 59 completed 5 or fewer.

Study procedures were approved by the University of Pittsburgh Institutional Review Board. All participants received monetary compensation for their participation.

\section{Assessments}


Demographic and clinical information. Participants self-reported demographic information including age, racial identity, annual household income, educational background, and parity at T0. Individuals were asked to describe ethnic identity as either Hispanic or Latina or not and to circle any of the following category or categories to best describe their race: White, Black or African American, Asian, American Indian/Alaska Native, Native Hawaiian or Other Pacific Islander. Given that well established disparity in the health of Black pregnant individuals relative to those of other racial identities (Ross, Guardino, Dunkel Schetter, \& Hobel, 2018; Thoma et al., 2019) we also compared individuals who identified as Black or African American relative to other racial identities in analyses involving demographic data. Participants also were queried about smoking and smoking status was coded as ever relative to never smoking

Pre-pregnancy weight. During the initial phone screen, participants self-reported prepregnancy weight, a commonly used, valid method of obtaining pre-pregnancy weight (Shin, Chung, Weatherspoon, \& Song, 2014). During the baseline (T0) assessment, height was measured in person via calibrated stadiometer and weight was measured using a digital scale. Prepregnancy BMI was calculated using self-reported pre-pregnancy weight obtained during the initial phone screen and measured height at the TO assessment.

Gestational weight gain. GWG was calculated as the measured weight before delivery as obtained from medical records minus self-reported pre-pregnancy weight obtained during the initial phone screen and defined as excessive according to IOM guidelines (Institute of Medicine \& Committee to Reexamine Pregnancy Weight Guidelines, 2009). Both the dichotomous variable representing GWG above IOM guidelines for pre-pregnancy BMI, and the total amount of GWG were used as dependent variables.

Medical record data. Because the length of gestation is a potential confounder of GWG, delivery dates were abstracted from maternal medical charts to calculate length of gestation. Data on medical conditions and diagnoses during pregnancy also were abstracted.

Eating Disorder Examination-Pregnancy Version. The Eating Disorder Examination-Pregnancy Version (EDE-PV) is a structured clinical interview designed to assess eating disorder psychopathology (Fairburn, Cooper, \& O'Connor, 1993) modified to capture the unique eating attitudes and behaviors associated with pregnancy (Emery et al., 2017). Like the EDE, (Fairburn et al., 1993) the EDE-PV assesses distinct types of overeating and LOC episodes, including objective bulimic episodes (OBEs; characterized by the consumption of an objectively large amount of food accompanied by a sense of LOC), subjective bulimic episodes (SBEs; an episode of LOC in which the amount of food consumed is not deemed objectively large), and overeating episodes in which LOC is endorsed that occur outside of OBEs and SBEs. As shown in Table 2, we defined any LOC as the experience of any type of LOC episode (i.e., OBE, SBE, or episodes of LOC outside of OBE or SBE).

Interviewers were extensively trained on the EDE-PV. Coding of episode size as well as the presence/absence of LOC episodes were discussed weekly and decisions on size and LOC were made by team consensus. At T0, participants were queried about the past three months of pregnancy as well as the three months prior to pregnancy, yielding two distinct measurement time periods. The presence ( $\geq 1$ LOC episode) or absence of LOC during these two 3-month periods was documented. At T0, interrater reliability for LOC episodes was high (intraclass correlation coefficient $=0.89)($ Kolko et al., 2017).

At monthly prenatal telephone assessments (T1-T5), participants were interviewed using a short version of the EDE-PV, comprised only of the overeating section. The presence ( $\geq 1$ LOC episode) or absence of LOC during each of these monthly periods was documented. Participants were additionally dichotomized according to whether they experienced LOC ( $\geq 1$ LOC episode) at any point during pregnancy (T0-T5).

\section{Analytic Plan}

As shown in Figure 1, participants were excluded from analysis due to stillbirth $(n=3)$ or an inability to obtain medical records $(n=7)$, resulting in an analytic sample of 247 participants. Descriptive statistics were calculated to characterize the 
sample and study aims were tested using linear and logistic regression. Given that age, racial identity, educational background, income, parity, and smoking status have been linked to GWG (Brawarsky et al., 2005; Olson \& Strawderman, 2003; Strychar et al., 2000), these variables were included as covariates in all models. Pre-pregnancy BMI also was included as a covariate in all models given the relationship between pre-pregnancy overweight or obesity and GWG. The length of gestation also is a potential confounder of GWG and was covaried in analyses of GWG.

Analyses were designed to test the association between LOC and total GWG as well as the likelihood of exceeding IOM guidelines for GWG. First, participants were categorized into four groups according to LOC patterns in the three months prior to pregnancy through the end of pregnancy: (a). No LOC prior to or during pregnancy $(61.5 \%)$; (b). LOC prior to pregnancy but not during (1.2\%); (c). LOC during pregnancy only (24.7\%); and (d). LOC both prior to and during pregnancy (12.6\%). Only 3 $(1.2 \%)$ of participants reported LOC prior to pregnancy but not during and these individuals were excluded from analysis to ensure adequate model fit. Linear regression models were fit to examine the relationship between the remaining three LOC patterns (never, both during and prior to pregnancy, and during pregnancy only) and total GWG, adjusting for the covariates listed above. Binary logistic regression analysis was used to analyze the association between LOC patterns and the likelihood of exceeding IOM guidelines for GWG, adjusting for covariate effects. Comparisons for the linear regression and logistic regression models were respectively tested via t-tests and Likelihood Ratio tests for the corresponding contrasts. We set a priori contrasts between LOC during pregnancy only and absence of LOC prior to or during pregnancy, and between LOC during pregnancy only and absence of LOC prior to or during pregnancy.

Next, we used number of LOC episodes in an additional model examining the relationship between LOC eating episodes in pregnancy and GWG. We fit a linear regression model to predict total GWG and a binary logistic regression model to predict the likelihood of exceeding IOM guidelines. Both models were adjusted for the covariates described previously. Because the number of LOC episodes was highly skewed, a log transformation was applied to episode data.

Finally, we evaluated the relationship between LOC eating and GWG in the context of obstetric complications that may impact GWG. Two complications were examined given their potential influence on weight gain: bed rest $(n=2)$ and diabetes diagnosed during this pregnancy $(n=15)$. Given the low frequency of bed rest $(0.8 \%$ of the analytic sample), this variable was not further evaluated as a covariate. Diabetes diagnosis during pregnancy was examined in models predicting both total GWG and excessive GWG along with the other covariates. All data were analyzed using SAS 9.4.

\section{Results}

\section{Rates of LOC}

Demographic and clinical characteristics are described in Table 1 as are differences in these characteristics and GWG between those who did and did not experience LOC during pregnancy. Table 2 shows the proportion of pregnant people assessed who experienced an episode of any LOC in the three months evaluated prior to pregnancy as well as in the 5 monthly periods across pregnancy. As shown, 38.5\% of pregnant people endorsed LOC prior to or during pregnancy and 3 pregnant people $(1.2 \%)$ reported LOC only prior to pregnancy.

\section{Any LOC as a predictor of total GWG}

After controlling for relevant covariates, pregnant people who reported LOC during pregnancy gained $3.14 \mathrm{~kg}$ more weight than did those who did not experience LOC prior to or during pregnancy ( $p=0.03$; See Table 3$)$. In contrast, participant who reported LOC both prior to and during pregnancy did not differ in their total GWG from those who did not report LOC prior to or during pregnancy $(B=-0.60,95 \% \mathrm{Cl}[-8.50,7.31], \mathrm{p}=0.88)$. In addition, nulliparous pregnant people gained $4.06 \mathrm{~kg}$ more during gestation than did multiparous pregnant people $(p=0.01)$. Also, each unit increase of pregnant people's prepregnancy BMI led to $0.2 \mathrm{~kg}$ decrease in GWG ( $p=0.04)$, after controlling for covariates.

\section{Any LOC as a predictor of excessive GWG}


Overall, 78.7\% $(n=48 / 61)$ of pregnant people who reported LOC during pregnancy exceeded IOM guidelines for GWG. After controlling for relevant covariates, pregnant people who endorsed LOC during pregnancy only $(n=61)$ were more likely to exceed IOM guidelines for GWG compared to those who did not endorse LOC prior to or during pregnancy (log odd ratio= $0.86,95 \% \mathrm{Cl}[0.11,1.66], \mathrm{p}=0.03$, see Table 3). Pregnant people who reported LOC prior to and during pregnancy $(n=31)$ did not differ in their likelihood of exceeding IOM recommendations for GWG compared to those who did not report LOC prior to or during pregnancy (log odd ratio $=-0.16,95 \% \mathrm{Cl}[-1.01,0.72], \mathrm{p}=0.71$ ).

In addition, pregnant people who were smoking at T0 (log odd ratio $=0.91,95 \% \mathrm{Cl}[0.08,1.81], \mathrm{p}=0.04)$ and nulliparous pregnant people (log odd ratio $=0.89,95 \% \mathrm{Cl}[0.21,1.61], \mathrm{p}=0.01$ ) were more likely to exceed IOM guidelines and gain excessive gestational weight compared to their non-smoking and multiparous counterparts. None of the other covariates were found to be significant in the models of LOC predicting GWG.

\section{Number of LOC episodes as a predictor of total and excessive GWG}

As shown in Table 4, among pregnant people who reported LOC in pregnancy, the log of the number of LOC episodes positively related to total GWG. Pregnant people with more frequent episodes of LOC had higher GWG even after including covariate effects than did those with fewer episodes $(2.17 \mathrm{~kg} ., 95 \% \mathrm{Cl}[0.52,3.82], \mathrm{p}=0.01)$. However, in this group of among who reported LOC in pregnancy, the log of number of LOC episodes was not significantly related to the likelihood of exceeding GWG after controlling for relevant covariates (log odd ratio $=0.30,95 \% \mathrm{Cl}[-0.11,0.74], \mathrm{p}=0.17$ ).

\section{LOC and GWG after controlling for diabetes diagnosed during pregnancy}

Finally, we evaluated the relative contribution of LOC to total GWG and excessive GWG, controlling for the diagnosis of diabetes during pregnancy in addition to the other covariates. Pregnant people who were diagnosed with diabetes during pregnancy $(n=15)$ began pregnancy with a BMI of $40.39( \pm 9.21) \mathrm{kg} / \mathrm{m}^{2}$, gained $22.04( \pm 12.12) \mathrm{kg}$ during pregnancy, and $87 \%(n=13 / 15)$ exceeded IOM guidelines for GWG. Among pregnant people diagnosed with diabetes during pregnancy, the $46.7 \%$ who reported at least one episode of LOC endorsed an average of $15.93( \pm 30.74)$ LOC episodes across pregnancy. A diagnosis of diabetes during pregnancy was significantly related to total GWG $(p<0.01)$ and marginally associated with excessive GWG ( $p=0.09)$. Importantly, LOC during pregnancy remained significantly associated with both total GWG $(p=$ 0.049) and excessive GWG ( $p=0.04)$ even after diabetes diagnosed during pregnancy was included as a covariate, though this effect was attenuated.

\section{Discussion}

Pregnant people with overweight or obesity prior to pregnancy who experience LOC at any point during but not prior to pregnancy gain substantially more gestational weight than do those who do not experience LOC. Specifically, pregnant people who report LOC during pregnancy but not in the three months prior to pregnancy gain over $3 \mathrm{~kg}$ more than do those who do not experience prenatal LOC. Compared to pregnant people who do not report LOC, pregnant people who report LOC during but not prior to pregnancy are 2.36 times more likely to gain an amount of weight that exceeds that recommended by the IOM based on their prepregnancy weight status. Moreover, prenatal LOC was linked to greater GWG after adjusting for prepregnancy BMI, a consistent and robust predictor of GWG. Thus, LOC is an identifiable behavioral phenotype and is associated with increased GWG among pregnant people with prepregnancy overweight or obesity. This finding linking LOC to GWG among pregnant people with prepregnancy overweight/obesity is consistent with recent results in a community sample of pregnant people (Micali et al., 2018). Micali and colleagues (2018) found that LOC was associated with higher GWG and rates of exceeding IOM GWG guidelines. The current data extend these findings and demonstrate that LOC at any point during pregnancy, is related to greater total GWG and increased likelihood of exceeding IOM recommended GWG amounts among pregnant people with prepregnancy overweight or obesity, a group at high risk for poor obstetric outcomes. 
Although LOC was uniquely related to GWG after controlling for factors that have been linked to GWG, parity was also independently related to total GWG, and parity, racial identity and smoking status affected the likelihood of exceeding IOM guidelines for GWG. Nulliparous pregnant people were more likely to have a higher total GWG and to exceed IOM guidelines for GWG compared to multiparous pregnant people. In addition, pregnant people who reported current smoking were more likely to exceed IOM guidelines for GWG relative to white pregnant people and those not currently smoking. Previous data suggest that pregnant people who experience LOC during pregnancy are more likely to be multiparous (Micali et al., 2018), although parity was not a significant predictor in previously reported associations between LOC and GWG (Hecht et al., 2020; Micali et al., 2018). Similarly, previous work has linked smoking cessation to increased GWG (Levine, Cheng, Cluss, Marcus, \& Kalarchian, 2013) though it is important to acknowledge that the $17 \%$ of pregnant people in this sample who reported smoking at baseline may have quit smoking during pregnancy.

Although racial identity was not a significant covariate in the models of LOC predicting GWG outcomes, substantial prior research has documented that pregnant people who identify as Black or African American are at increased risk for adverse obstetric outcomes relative to white pregnant people, in part due to systemic factors such as structural racism that impact access to and quality of prenatal care (Bentley-Lewis et al., 2014; Karlamangla et al., 2005; Ross et al., 2018; Thoma et al., 2019). Relatedly, we have previously documented that rates of LOC during pregnancy only are endorsed by those who identify as Black or African American (Donofry et al., 2021). Thus, LOC may be a potential target for interventions designed to improve Black pregnant people's perinatal health.

The finding that LOC during pregnancy was independently associated with higher GWG and increased likelihood of exceeding IOM recommendations, suggests the importance of assessing LOC throughout pregnancy. There also are important clinical implications. First, LOC is a potentially modifiable factor that can be addressed during pregnancy. Evidence-based interventions for LOC have been established in non-pregnant individuals, including among those with overweight or obesity (Grilo, Masheb, Wilson, Gueorguieva, \& White, 2011; Thoma et al., 2019). Effective interventions to reduce LOC differ from general weight control interventions and indicate the need to address the psychopathology related to LOC. Previous studies have shown that LOC is prevalent among pregnant people with overweight or obesity, related to weight gain and modifiable. Our results extend these reports and demonstrate that LOC predicts weight gain among pregnant people with overweight or obesity during pregnancy above and beyond demographic and pregnancy-related covariates.

Second, addressing LOC during pregnancy may have important benefits to the offspring of pregnant people who endorse LOC. LOC during pregnancy has been found to predict subsequent eating and weight outcomes among infants and children (Micali et al., 2018). Potential mechanisms for this link include biological pathways related to fetal programming, maternal dietary intake during pregnancy (Micali et al., 2018), as well as the influence of genetic, epigenetic, and postpartum environmental factors. For example, we previously reported that mothers' LOC during pregnancy was prospectively associated with obesity-related eating patterns in their infants (Kolko, Salk, Sweeny, Marcus, \& Levine, 2018). Thus, helping pregnant people to manage LOC and eating patterns during pregnancy may have significant benefits for infants and children. Third, it is possible that interventions designed to address LOC will affect not only GWG but also other common perinatal mental health outcomes. Indeed, our work (Kolko et al., 2017) and that of others (Hecht et al., 2020) documents a link between LOC and perinatal depressive symptoms, and it may be that depressive symptoms are a more robust predictor of GWG than self-reported eating patterns (Hecht et al., 2020).

These data are the first from a study specifically designed to prospectively evaluate the relationship between eating patterns and weight gain among pregnant people at high risk for excessive GWG. In addition, the present study repeated measurements of LOC with a semi-structured interview adapted for administration during pregnancy and data were collected on large and diverse sample of pregnant people. Moreover, the results considered the contribution of LOC to GWG after adjusting for key covariates. Nonetheless there are important limitations. The study is restricted by the exclusion of pregnant people who do not have prepregnancy overweight or obesity. Additionally, LOC prior to pregnancy was retrospectively characterized at a baseline study visit in the first half of pregnancy. Longitudinal studies that document pregnant people's 
LOC and weight trajectories prior to becoming pregnant are warranted to improve our understanding of the course of LOC and its relationship with weight prior to, during, and after pregnancy.

\section{Conclusion}

In summary, results of this longitudinal assessment of eating behavior among pregnant people at high risk for negative obstetric and later health outcomes, suggest that LOC during pregnancy is a predictor of GWG and predicts GWG beyond other known correlates of excessive gestations weight gain. LOC during pregnancy predicted both higher total GWG and a greater likelihood of exceeding IOM recommendations for GWG. Together, these results highlight the importance of addressing LOC among pregnant people and evaluating interventions for LOC during pregnancy.

\section{Abbreviations}

Gestational Weight Gain (GWG), Loss of Control eating (LOC), Body Mass Index (BMI), Institute of Medicine (IOM), Timepoint 0/Baseline (T0), Eating Disorder Examination (EDE), Eating Disorder Examination-Pregnancy Version (EDE-PV), Objective bulimic episodes (OBEs), Subjective bulimic episodes (SBEs).

\section{Declarations}

Ethics approval and consent to participate: This research has been reviewed and approved by the University of Pittsburgh Institutional Review Board. Informed consent to participate in the study was obtained prior to data collection from all participants.

Consent for Publication: Not applicable.

Available of data and material: Data described in the manuscript, code book, and analytic code will be made available upon request

Competing Interest: The authors have no conflicts of interest to declare.

Funding: This work was supported by National Institute of Child Health and Human Development [grant number R01HD068802]. Rebecca Emery's time was supported by National Center for Advancing Translational Sciences [grant numbers TL1R002493 and UL1TR002494].

Authors' contributions: Author contributions, but initials are as follows: MDL designed research; MDL, RLE, RPKC, JMS, GMS conducted research; BW, YC, RLE analyzed data; MDL, RLE, RPKC, BW, YC wrote the paper; MDL had primary responsibility for final content. All authors read and approved the final manuscript.

Acknowledgements: The authors wish to acknowledge with gratitude the pregnant people who participated in the interviews for this study and the capable assistance of Miriam Sheynblyum.

\section{References}

1. Amorim, A. R., Rössner, S., Neovius, M., Lourenço, P. M., \& Linné, Y. (2007). Does excess pregnancy weight gain constitute a major risk for increasing long-term BMI? Obesity (Silver Spring), 15(5), 1278-1286. doi:

10.1038/oby.2007.149

2. Bentley-Lewis, R., Powe, C., Ankers, E., Wenger, J., Ecker, J., \& Thadhani, R. (2014). Effect of race/ethnicity on hypertension risk subsequent to gestational diabetes mellitus. Am J Cardiol, 113(8), 1364-1370. https://doi.org/10.1016/j.amjcard.2014.01.411 
3. Blais, M. A., Becker, A. E., Burwell, R. A., Flores, A. T., Nussbaum, K. M., Greenwood, D. N., Herzog, D. B. (2000). Pregnancy: Outcome and impact on symptomatology in a cohort of eating-disordered women. Int J Eat Disord, 27(2), 140-149. doi: 10.1002/(sici)1098-108x(200003)27:2<140::aid-eat2>3.0.co;2-e

4. Brawarsky, P., Stotland, N. E., Jackson, R. A., Fuentes-Afflick, E., Escobar, G. J., Rubashkin, N., \& Haas, J. S. (2005). Prepregnancy and pregnancy-related factors and the risk of excessive or inadequate gestational weight gain. Int $J$ Gynaecol Obstet, 91(2), 125-131. doi: 10.1016/j.ijgo.2005.08.008

5. Bulik, C. M., Von Holle, A., Hamer, R., Knoph Berg, C., Torgersen, L., Magnus, P., Reichborn-Kjennerud, T. (2007). Patterns of remission, continuation and incidence of broadly defined eating disorders during early pregnancy in the Norwegian Mother and Child Cohort Study (MoBa). Psychol Med, 37(8), 1109-1118. doi: 10.1017/s0033291707000724

6. Chen, H. Y., \& Chauhan, S. P. (2019). Association between gestational weight gain adequacy and adverse maternal and neonatal outcomes. Am J Perinatol, 36(6), 615-623. doi: 10.1055/s-0038-1672196

7. Colles, S. L., Dixon, J. B., \& O'Brien, P. E. (2008). Loss of control is central to psychological disturbance associated with binge eating disorder. Obesity (Silver Spring), 16(3), 608-614. doi: 10.1038/oby.2007.99

8. Crow, S. J., Agras, W. S., Crosby, R., Halmi, K., \& Mitchell, J. E. (2008). Eating disorder symptoms in pregnancy: A prospective study. Int J Eat Disord, 41(3), 277-279. doi: 10.1002/eat.20496

9. Donofry, S. D., Emery, R. L., Kolko Conlon, R. P., Germeroth, L. J., Wang, B., Cheng, Y., Levine, M. D. (2021). Documenting the course of loss of control over eating prior to, during and after pregnancy among women with pre-pregnancy overweight and obesity. Int J Eat Disord, 54(4), 633-638. doi: 10.1002/eat.23454

10. Easter, A., Bye, A., Taborelli, E., Corfield, F., Schmidt, U., Treasure, J., \& Micali, N. (2013). Recognising the symptoms: how common are eating disorders in pregnancy? Eur Eat Disord Rev, 21(4), 340-344. doi: 10.1002/erv.2229

11. Emery, R. L., Grace, J. L., Kolko, R. P., \& Levine, M. D. (2017). Adapting the eating disorder examination for use during pregnancy: Preliminary results from a community sample of women with overweight and obesity. Int J Eat Disord, 50(5), 597-601. doi: 10.1002/eat.22646

12. Engel, S. G., Kahler, K. A., Lystad, C. M., Crosby, R. D., Simonich, H. K., Wonderlich, S. A., Mitchell, J. E. (2009). Eating behavior in obese BED, obese non-BED, and non-obese control participants: a naturalistic study. Behav Res Ther, 47(10), 897-900. doi: 10.1016/j.brat.2009.06.018

13. Fairburn, C. G., Cooper, Z., \& O’Connor, M. (1993). The eating disorder examination. Int J Eat Disord, 6, 1-8.

14. Goldstein, R. F., Abell, S. K., Ranasinha, S., Misso, M., Boyle, J. A., Black, M. H., Teede, H. J. (2017). Association of gestational weight gain with maternal and infant outcomes: A systematic review and meta-analysis. JAMA, 317(21), 2207-2225. doi: 10.1001/jama.2017.3635

15. Gorin, A. A., Niemeier, H. M., Hogan, P., Coday, M., Davis, C., DiLillo, V. G., Yanovski, S. Z. (2008). Binge eating and weight loss outcomes in overweight and obese individuals with type 2 diabetes: results from the Look AHEAD trial. Arch Gen Psychiatry, 65(12), 1447-1455. doi: 10.1001/archpsyc.65.12.1447

16. Grilo, C. M., Masheb, R. M., Wilson, G. T., Gueorguieva, R., \& White, M. A. (2011). Cognitive-behavioral therapy, behavioral weight loss, and sequential treatment for obese patients with binge-eating disorder: A randomized controlled trial. $J$ Consult Clin Psychol, 79(5), 675-685. doi: 10.1037/a0025049

17. Hecht, L. M., Schwartz, N., Miller-Matero, L. R., Braciszewski, J. M., \& Haedt-Matt, A. (2020). Eating pathology and depressive symptoms as predictors of excessive weight gain during pregnancy. J Health Psychol, 1359105320913934. doi: $10.1177 / 1359105320913934$

18. Heery, E., Wall, P. G., Kelleher, C. C., \& McAuliffe, F. M. (2016). Effects of dietary restraint and weight gain attitudes on gestational weight gain. Appetite, 107, 501-510. doi: 10.1016/j.appet.2016.08.103

19. Institute of Medicine (US), National Research Council (US),Committee to Reexamine IOM Pregnancy Weight Guidelines. (2009). Weight Gain during pregnancy: Reexamining the guidelines. National Academies Press, Washington (DC). https://doi.org/10.17226/12584 
20. Karlamangla, A. S., Singer, B. H., Williams, D. R., Schwartz, J. E., Matthews, K. A., Kiefe, C. I., \& Seeman, T. E. (2005). Impact of socioeconomic status on longitudinal accumulation of cardiovascular risk in young adults: The CARDIA Study (USA). Soc Sci Med, 60(5), 999-1015. doi: 10.1016/j.socscimed.2004.06.056

21. Kolko, R. P., Emery, R. L., Marcus, M. D., \& Levine, M. D. (2017). Loss of control over eating before and during early pregnancy among community women with overweight and obesity. Int J Eat Disord, 50(5), 582-586. doi: 10.1002/eat.22630

22. Kolko, R. P., Salk, R. H., Sweeny, G. M., Marcus, M. D., \& Levine, M. D. (2018). Mothers' loss of control over eating during pregnancy in relation to their infants' appetitive traits. Appetite, 120, 1-5. doi: 10.1016/j.appet.2017.08.007

23. Latner, J. D., Hildebrandt, T., Rosewall, J. K., Chisholm, A. M., \& Hayashi, K. (2007). Loss of control over eating reflects eating disturbances and general psychopathology. Behav Res Ther, 45(9), 2203-2211. doi: 10.1016/j.brat.2006.12.002

24. Levine, M. D., Cheng, Y., Cluss, P. A., Marcus, M. D., \& Kalarchian, M. A. (2013). Prenatal smoking cessation intervention and gestational weight gain. Women's Health Issues, 23(6), e389-e393.

25. Linné, Y., Dye, L., Barkeling, B., \& Rössner, S. (2004). Long-term weight development in women: a 15-year follow-up of the effects of pregnancy. Obes Res, 12(7), 1166-1178. doi: 10.1038/oby.2004.146

26. Marcus, M. D., \& Wildes, J. E. (2009). Obesity: Is it a mental disorder? Int J Eat Disord, 42(8), 739-753. doi: 10.1002/eat.20725

27. Micali, N., Al Essimii, H., Field, A. E., \& Treasure, J. (2018). Pregnancy loss of control over eating: A longitudinal study of maternal and child outcomes. Am J Clin Nutr, 108(1), 101-107. doi: 10.1093/ajcn/nqy040

28. Morgan, J. F., Lacey, J. H., \& Chung, E. (2006). Risk of postnatal depression, miscarriage, and preterm birth in bulimia nervosa: Retrospective controlled study. Psychosom Med, 68(3), 487-492. doi: 10.1097/01.psy.0000221265.43407.89

29. Nunes, M. A. A., Pinheiro, A. P., Camey, S. A., \& Schmidt, M. I. (2012). Binge eating during pregnancy and birth outcomes: A cohort study in a disadvantaged population in Brazil. Int J Eat Disord, 45(7), 827-831.

30. Olson, C. M., \& Strawderman, M. S. (2003). Modifiable behavioral factors in a biopsychosocial model predict inadequate and excessive gestational weight gain. J Am Diet Assoc, 103(1), 48-54. doi: 10.1053/jada.2003.50001

31. Park, C. K., Krebs, L., Lutsiv, O., van Blyderveen, S., Schmidt, L. A., Beyene, J., McDonald, S. D. (2015). Binge eating predicts excess gestational weight gain: A pilot prospective cohort study. J Obstet Gynaecol Can, 37(6), 494-507. doi: https://doi.org/10.1016/S1701-2163(15)30226-7

32. Rosenberg, T. J., Garbers, S., Chavkin, W., \& Chiasson, M. A. (2003). Prepregnancy weight and adverse perinatal outcomes in an ethnically diverse population. Obstet Gynecol, 102(5 Pt 1), 1022-1027. doi:

10.1016/j.obstetgynecol.2003.07.005

33. Ross, K. M., Guardino, C., Dunkel Schetter, C., \& Hobel, C. J. (2018). Interactions between race/ethnicity, poverty status, and pregnancy cardio-metabolic diseases in prediction of postpartum cardio-metabolic health. Ethn Health, 25, 11451160. https://doi.org/10.1080/13557858.2018.1493433

34. Schieve, L. A., Cogswell, M. E., \& Scanlon, K. S. (1998). An empiric evaluation of the Institute of Medicine's pregnancy weight gain guidelines by race. Obstet Gynecol, 91(6), 878-884. doi: 10.1016/s0029-7844(98)00106-9

35. Shin, D., Chung, H., Weatherspoon, L., \& Song, W. O. (2014). Validity of prepregnancy weight status estimated from selfreported height and weight. Matern Child Health J, 18(7), 1667-1674. doi: 10.1007/s10995-013-1407-6

36. Soares, R. M., Nunes, M. A., Schmidt, M. I., Giacomello, A., Manzolli, P., Camey, S., Duncan, B. B. (2009). Inappropriate eating behaviors during pregnancy: Prevalence and associated factors among pregnant women attending primary care in southern Brazil. Int J Eat Disord, 42(5), 387-393. doi: 10.1002/eat.20643

37. Striegel-Moore, R. H., Rosselli, F., Perrin, N., DeBar, L., Wilson, G. T., May, A., Kraemer, H. C. (2009). Gender difference in the prevalence of eating disorder symptoms. Int J Eat Disord, 42(5), 471-474. doi: 10.1002/eat.20625

38. Strychar, I. M., Chabot, C., Champagne, F., Ghadirian, P., Leduc, L., Lemonnier, M. C., Raynauld, P. (2000). Psychosocial and lifestyle factors associated with insufficient and excessive maternal weight gain during pregnancy. J Am Diet 
Assoc, 100(3), 353-356. doi: 10.1016/s0002-8223(00)00107-3

39. Swann, R. A., Von Holle, A., Torgersen, L., Gendall, K., Reichborn-Kjennerud, T., \& Bulik, C. M. (2009). Attitudes toward weight gain during pregnancy: Results from the Norwegian Mother and Child Cohort Study (MoBa). Int J Eat Disord, 42(5), 394-401. doi: 10.1002/eat.20632

40. Tang, X., Andres, A., West, D. S., Lou, X., \& Krukowski, R. A. (2020). Eating behavior and weight gain during pregnancy. Eat Behav, 36, 101364. doi: 10.1016/j.eatbeh.2020.101364

41. Thoma, M. E., Drew, L. B., Hirai, A. H., Kim, T. Y., Fenelon, A., Shenassa, E. D. (2019). Black-White disparities in preterm birth: Geographic, social, and health determinants. Am J Prev Med, 57(5), 675-686. doi: 10.1016/j.amepre.2019.07.007

42. Vesco, K. K., Dietz, P. M., Rizzo, J., Stevens, V. J., Perrin, N. A., Bachman, D. J., Hornbrook, M. C. (2009). Excessive gestational weight gain and postpartum weight retention among obese women. Obstet Gynecol, 114(5), 1069-1075. doi: 10.1097/AOG.0b013e3181baeacf

43. Yanovski, S. Z., Leet, M., Yanovski, J. A., Flood, M., Gold, P. W., Kissileff, H. R., Walsh, B. T. (1992). Food selection and intake of obese women with binge-eating disorder. Am J Clin Nutr, 56(6), 975-980. doi: 10.1093/ajcn/56.6.975

\section{Tables}

Table 1. Sample Characteristics $(\mathrm{N}=247)$ 


\begin{tabular}{|c|c|c|c|c|}
\hline \multirow{2}{*}{$\begin{array}{l}\text { Variable } \\
\text { LOC Patterns }\end{array}$} & \multicolumn{4}{|c|}{ Mean \pm SD or $\%(n)$} \\
\hline & All & No LOC & LOCP2P\&Preg & LOCPregOnly \\
\hline Age, years & $28.53 \pm 5.42$ & $28.42 \pm 5.25$ & $28.86 \pm 5.87$ & $28.74 \pm 5.79$ \\
\hline \multicolumn{5}{|l|}{ Racial identity } \\
\hline Black/African American & $45.8 \%(113)$ & $28.7 \%(71)$ & $6.5 \%(16)$ & $17 \%(42)$ \\
\hline Others & $54.2 \%(134)$ & $36 \%(89)$ & $8.1 \%(20)$ & $9.7 \%(24)$ \\
\hline \multicolumn{5}{|l|}{ Parity } \\
\hline Nulliparous & $34.8 \%(86)$ & $24.7 \%(61)$ & $4 \%(10)$ & $5.7 \%(14)$ \\
\hline Multiparous & $65.2 \%(161)$ & $36.8 \%(91)$ & $8.5 \%(21)$ & $19 \%(47)$ \\
\hline \multicolumn{5}{|l|}{ Education } \\
\hline$\leq$ High School Degree & $33.2 \%(82)$ & $17 \%(42)$ & $4.5 \%(11)$ & $11.3 \%(28)$ \\
\hline > High School Degree & $66.8 \%(165)$ & $44.5 \%(110)$ & $8.1 \%(20)$ & $13.4 \%(33)$ \\
\hline \multicolumn{5}{|l|}{ Annual Household Income } \\
\hline$\leq \$ 30000$ & $65.6 \%(162)$ & $36.4 \%(90)$ & $8.1 \%(20)$ & $20.2 \%(50)$ \\
\hline$>\$ 30000$ & $34.4 \%(85)$ & $25.1 \%(62)$ & $4.5 \%(11)$ & $4.5 \%(11)$ \\
\hline \multicolumn{5}{|l|}{ Smoking } \\
\hline No & $83 \%(205)$ & $51.8 \%(128)$ & $9.7 \%(24)$ & $20.2 \%(50)$ \\
\hline Yes & $17 \%(42)$ & $9.7 \%(24)$ & $2.8 \%(7)$ & $4.5 \%(11)$ \\
\hline Pre-pregnancy weight, pounds & $197 \pm 42.34$ & $193.97 \pm 40.77$ & $201.45 \pm 45.1$ & $201.61 \pm 44.84$ \\
\hline Pre-pregnancy BMI, kg/m2 & $32.78 \pm 6.35$ & $32.34 \pm 6.14$ & $33.97 \pm 6.66$ & $33.2 \pm 6.78$ \\
\hline \multicolumn{5}{|l|}{ Pre-pregnancy Weight Status } \\
\hline Overweight & $42.5 \%(105)$ & $27.5 \%(68)$ & $4.9 \%(12)$ & $9.7 \%(24)$ \\
\hline Obese & $57.5 \%(142)$ & $34 \%(84)$ & $7.7 \%(19)$ & $15 \%(37)$ \\
\hline GWG, Ibs & $13.47 \pm 9.34$ & $12.79 \pm 8.8$ & $12.56 \pm 10.26$ & $15.88 \pm 10.05$ \\
\hline \multicolumn{5}{|l|}{ GWG Category } \\
\hline Inadequate & $16.6 \%(41)$ & $10.9 \%(27)$ & $2.4 \%(6)$ & $3.2 \%(8)$ \\
\hline Adequate & $15.4 \%(38)$ & $10.1 \%(25)$ & $2.4 \%(6)$ & $2 \%(5)$ \\
\hline Excessive & $68.02 \%(168)$ & $40.5 \%(100)$ & $7.7 \%(19)$ & $19.4 \%(48)$ \\
\hline Gestational length, weeks & 38.67 & 38.38 & 39.55 & 38.97 \\
\hline \multicolumn{5}{|c|}{ Note: $\mathrm{BMI}=$ body mass index; GWG = gestational weight gain } \\
\hline
\end{tabular}

Table 2. Sample mean (sample standard deviation) of LOC episodes and rates (count) by LOC types in the three months prior to and during pregnancy 


\begin{tabular}{|c|c|c|c|c|c|c|c|c|c|}
\hline $\begin{array}{l}\text { Type of } \\
\text { LOCITime } \\
\text { assessments }\end{array}$ & Statistics & $\begin{array}{l}\text { P2P } \\
\text { (3 months) }\end{array}$ & $\begin{array}{l}\text { T0 } \\
\text { (3 months) }\end{array}$ & $\begin{array}{l}\text { T1 } \\
\text { (1 } \\
\text { month) }\end{array}$ & $\begin{array}{l}\text { T2 } \\
\text { (1 } \\
\text { month) }\end{array}$ & $\begin{array}{l}\text { T3 } \\
\text { (1 } \\
\text { month) }\end{array}$ & $\begin{array}{l}\text { T4 } \\
\text { (1 } \\
\text { month) }\end{array}$ & $\begin{array}{l}\text { T5 } \\
\text { (1 } \\
\text { month) }\end{array}$ & All \\
\hline \multirow[t]{2}{*}{ OBE } & $\begin{array}{l}\text { Mean } \\
\text { (SD) }\end{array}$ & $\begin{array}{l}1.56 \\
(10.56)\end{array}$ & $1.49(8.66)$ & $\begin{array}{l}0.28 \\
(1.91)\end{array}$ & $\begin{array}{l}0.18 \\
(1.19)\end{array}$ & $\begin{array}{l}0.1 \\
(0.56)\end{array}$ & $\begin{array}{l}0.18 \\
(1.41)\end{array}$ & $\begin{array}{l}0.06 \\
(0.32)\end{array}$ & $\begin{array}{l}3.81 \\
(16.32)\end{array}$ \\
\hline & $\%(n)$ & $6.9 \%(17)$ & $10.1 \%(15)$ & $\begin{array}{l}6.9 \% \\
(17)\end{array}$ & $\begin{array}{l}7.3 \% \\
(18)\end{array}$ & $\begin{array}{l}4.5 \% \\
(11)\end{array}$ & $\begin{array}{l}5.3 \% \\
(13)\end{array}$ & $\begin{array}{l}2.8 \% \\
(7)\end{array}$ & $\begin{array}{l}23.9 \% \\
(59)\end{array}$ \\
\hline \multirow[t]{2}{*}{ SBE } & $\begin{array}{l}\text { Mean } \\
\text { (SD) }\end{array}$ & $\begin{array}{l}1.96 \\
(12.38)\end{array}$ & $1.61(10.5)$ & $\begin{array}{l}0.3 \\
(2.14)\end{array}$ & $\begin{array}{l}0.27 \\
(1.36)\end{array}$ & $\begin{array}{l}0.18 \\
(0.88)\end{array}$ & $\begin{array}{l}0.12 \\
(0.81)\end{array}$ & $\begin{array}{l}0.21 \\
(1.9)\end{array}$ & $\begin{array}{l}4.58 \\
(17.94)\end{array}$ \\
\hline & $\%(n)$ & $8.1 \%(20)$ & $15.4 \%(38)$ & $\begin{array}{l}7.7 \% \\
(19)\end{array}$ & $\begin{array}{l}7.3 \% \\
(18)\end{array}$ & $\begin{array}{l}6.9 \% \\
(17)\end{array}$ & $\begin{array}{l}4 \% \\
(10)\end{array}$ & $\begin{array}{l}2.8 \% \\
(7)\end{array}$ & $\begin{array}{l}28.3 \% \\
(70)\end{array}$ \\
\hline \multirow[t]{2}{*}{ OBE/SBE } & $\begin{array}{l}\text { Mean } \\
\text { (SD) }\end{array}$ & $3.52(20.29)$ & 3.1(13.99) & $\begin{array}{l}0.58 \\
(3.47)\end{array}$ & $\begin{array}{l}0.45 \\
(1.82)\end{array}$ & $\begin{array}{l}0.28 \\
(1.02)\end{array}$ & $\begin{array}{l}0.3 \\
(1.61)\end{array}$ & $\begin{array}{l}0.27 \\
(1.92)\end{array}$ & $\begin{array}{l}8.4 \\
(28.58)\end{array}$ \\
\hline & $\%(n)$ & $13.4 \%(33)$ & $22.7 \%(56)$ & $\begin{array}{l}12.1 \% \\
(30)\end{array}$ & $\begin{array}{l}12.6 \% \\
(31)\end{array}$ & $\begin{array}{l}11.3 \% \\
(28)\end{array}$ & $\begin{array}{l}9.3 \% \\
(23)\end{array}$ & $\begin{array}{l}5.3 \% \\
(13)\end{array}$ & $\begin{array}{l}37.2 \% \\
(92)\end{array}$ \\
\hline \multirow{2}{*}{$\begin{array}{l}\text { LOC Outside } \\
\text { of OBE or } \\
\text { SBE Episode }\end{array}$} & $\begin{array}{l}\text { Mean } \\
\text { (SD) }\end{array}$ & $0.02(0.21)$ & $0.06(0.65)$ & $\begin{array}{l}0.04 \\
(0.44)\end{array}$ & $0(0)$ & $\begin{array}{l}0.02 \\
(0.16)\end{array}$ & $\begin{array}{l}0.03 \\
(0.43)\end{array}$ & $\begin{array}{l}0.01 \\
(0.08)\end{array}$ & $\begin{array}{l}0.13 \\
(0.95)\end{array}$ \\
\hline & $\%(n)$ & $0.4 \%(1)$ & $1.2 \%(3)$ & $\begin{array}{l}0.8 \% \\
(2)\end{array}$ & $0 \%(0)$ & $\begin{array}{l}0.8 \% \\
(2)\end{array}$ & $\begin{array}{l}0.4 \% \\
(1)\end{array}$ & $\begin{array}{l}0.4 \% \\
(1)\end{array}$ & $\begin{array}{l}3.6 \% \\
(9)\end{array}$ \\
\hline \multirow[t]{2}{*}{ Any LOC } & $\begin{array}{l}\text { Mean } \\
\text { (SD) }\end{array}$ & $3.53(20.29)$ & $3.14(13.99)$ & $\begin{array}{l}0.61 \\
(3.49)\end{array}$ & $\begin{array}{l}0.45 \\
(1.82)\end{array}$ & $\begin{array}{l}0.29 \\
(1.03)\end{array}$ & $\begin{array}{l}0.32 \\
(1.75)\end{array}$ & $\begin{array}{l}0.27 \\
(1.92)\end{array}$ & $\begin{array}{l}8.52 \\
(28.73)\end{array}$ \\
\hline & $\%(n)$ & $13.8 \%(34)$ & $23.5 \%(58)$ & $\begin{array}{l}13 \% \\
(32)\end{array}$ & $\begin{array}{l}12.6 \% \\
(31)\end{array}$ & $\begin{array}{l}12.1 \% \\
(30)\end{array}$ & $\begin{array}{l}9.3 \% \\
(23)\end{array}$ & $\begin{array}{l}5.7 \% \\
(14)\end{array}$ & $\begin{array}{l}38.5 \% \\
(95)\end{array}$ \\
\hline
\end{tabular}

Table 3. Relationship of loss of control eating to total and excessive gestational weight gain 


\begin{tabular}{|c|c|c|c|c|}
\hline & Total GWG, kgs & & Excessive GWG & \\
\hline Parameter & Estimate [95\% Cl] & $\mathrm{p}$ & Estimate [95\% Cl] & $\mathrm{p}$ \\
\hline Intercept & $-1.24[-23.99,21.51]$ & 0.91 & $-0.74[-6.31,4.80]$ & 0.79 \\
\hline \multicolumn{5}{|l|}{ LOC Group } \\
\hline No LOC (ref) & - & - & - & - \\
\hline LOC prior to and during pregnancy & $-0.27[-3.88,3.33]$ & 0.88 & $-0.16[-1.01,0.72]$ & 0.71 \\
\hline LOC in pregnancy only & $3.14[0.28,6]$ & 0.03 & $0.86[0.11,1.66]$ & 0.03 \\
\hline Age, years & $0.2[-0.05,0.45]$ & 0.11 & $0.04[-0.18,0.11]$ & 0.17 \\
\hline \multicolumn{5}{|l|}{ Racial identity } \\
\hline Black (ref) & - & - & - & - \\
\hline Others & $-2.09[-4.96,0.78]$ & 0.15 & $-0.34[-1.05,0.36]$ & 0.34 \\
\hline \multicolumn{5}{|l|}{ Education } \\
\hline > High School Degree (ref) & - & - & - & - \\
\hline$\leq$ High School Degree & $-0.02[-2.92,2.87]$ & 0.99 & $-0.34[-1.47,0.36]$ & 0.35 \\
\hline \multicolumn{5}{|l|}{ Annual Household Income } \\
\hline$>\$ 30000$ (ref) & - & - & - & - \\
\hline$\leq \$ 30000$ & $0.27[-3.18,3.71]$ & 0.88 & $-0.03[-0.89,0.82]$ & 0.94 \\
\hline \multicolumn{5}{|l|}{ Parity } \\
\hline Multiparous (ref) & - & - & - & - \\
\hline Nulliparous & $4.05[1.33,6.77]$ & $<0.01$ & $0.89[0.21,1.61]$ & 0.01 \\
\hline \multicolumn{5}{|l|}{ Smoking Status at Baseline } \\
\hline Not smoking (ref) & - & - & - & - \\
\hline Smoking & $0.82[-2.46,4.1]$ & 0.62 & $0.91[0.08,1.81]$ & 0.04 \\
\hline Pre-pregnancy BMI & $-0.2[-0.4,-0.01]$ & 0.04 & $-0.04[-0.85,0.01]$ & 0.12 \\
\hline Gestational length, weeks & $0.37[-0.11,0.84]$ & 0.13 & $0.03[-0.08,0.15]$ & 0.59 \\
\hline \multicolumn{5}{|c|}{$\begin{array}{l}\text { Note: The model examining total GWG was tested using multiple linear regression whereas the model examining the } \\
\text { likelihood of gaining excessive GWG was tested using binary logistic regression in reference to those who did not exceed } \\
\text { Institute of Medicine recommendations for GWG. Cl = confidence interval, LOC = loss of control eating, GWG = } \\
\text { gestational weight gain }\end{array}$} \\
\hline
\end{tabular}

Table 4. Regression models of relationship of loss of control eating episodes to total and excessive gestational weight gain 


\begin{tabular}{|c|c|c|c|c|}
\hline & Total GWG, kgs & & Excessive GWG & \\
\hline Parameter & Estimate [95\% Cl] & $\mathrm{p}$ & Estimate $[95 \% \mathrm{Cl}]$ & $\mathrm{p}$ \\
\hline Intercept & $-3.97[-57.63,49.67]$ & 0.88 & $-0.84[-13.37,11.68]$ & 0.89 \\
\hline LOC episodes, log-transformed & $2.17[0.49,3.85]$ & 0.01 & $0.30[-0.11,0.74]$ & 0.17 \\
\hline Age, years & $0.33[-0.15,0.80]$ & 0.17 & $0.04[-0.07,0.16]$ & 0.53 \\
\hline \multicolumn{5}{|l|}{ Racial identity } \\
\hline Black (ref) & - & - & - & - \\
\hline Others & $-1.07[-6.45,1.31]$ & 0.69 & $0.07[-1.14,1.34]$ & 0.91 \\
\hline \multicolumn{5}{|l|}{ Education } \\
\hline > High School Degree (ref) & - & - & - & - \\
\hline$\leq$ High School Degree & $3.43[-1.62,8.49]$ & 0.18 & $0.50[-0.68,1.70]$ & 0.41 \\
\hline \multicolumn{5}{|l|}{ Annual Household Income } \\
\hline$>\$ 30000$ (ref) & - & - & - & - \\
\hline$\leq \$ 30000$ & $2.96[-4.19,10.11]$ & 0.41 & $0.41[-1.28,2.14]$ & 0.64 \\
\hline \multicolumn{5}{|l|}{ Parity } \\
\hline Multiparous (ref) & - & - & - & - \\
\hline Nulliparous & $4.6[-0.52,9.74]$ & 0.08 & $0.36[-0.85,1.68]$ & 0.58 \\
\hline \multicolumn{5}{|l|}{ Smoking Status at Baseline } \\
\hline Not smoking (ref) & - & - & - & - \\
\hline Smoking & $-2.21[-7.97,3.55]$ & 0.45 & $-0.20[-1.57,1.28]$ & 0.78 \\
\hline Pre-pregnancy BMI & $-0.29[-0.62,0.03]$ & 0.08 & $-0.07[-0.14,0.01]$ & 0.08 \\
\hline Gestational length, weeks & $0.29[-0.91,1.5]$ & 0.63 & $0.05[-0.23,0.33]$ & 0.72 \\
\hline \multicolumn{5}{|c|}{$\begin{array}{l}\text { Note: The model examining total GWG was tested using multiple linear regression whereas the model examining the } \\
\text { likelihood of gaining excessive GWG was tested using binary logistic regression in reference to those who did not exceed } \\
\text { Institute of Medicine recommendations for GWG. CI = confidence interval, LOC = loss of control eating, GWG = } \\
\text { gestational weight gain }\end{array}$} \\
\hline
\end{tabular}

\section{Figures}




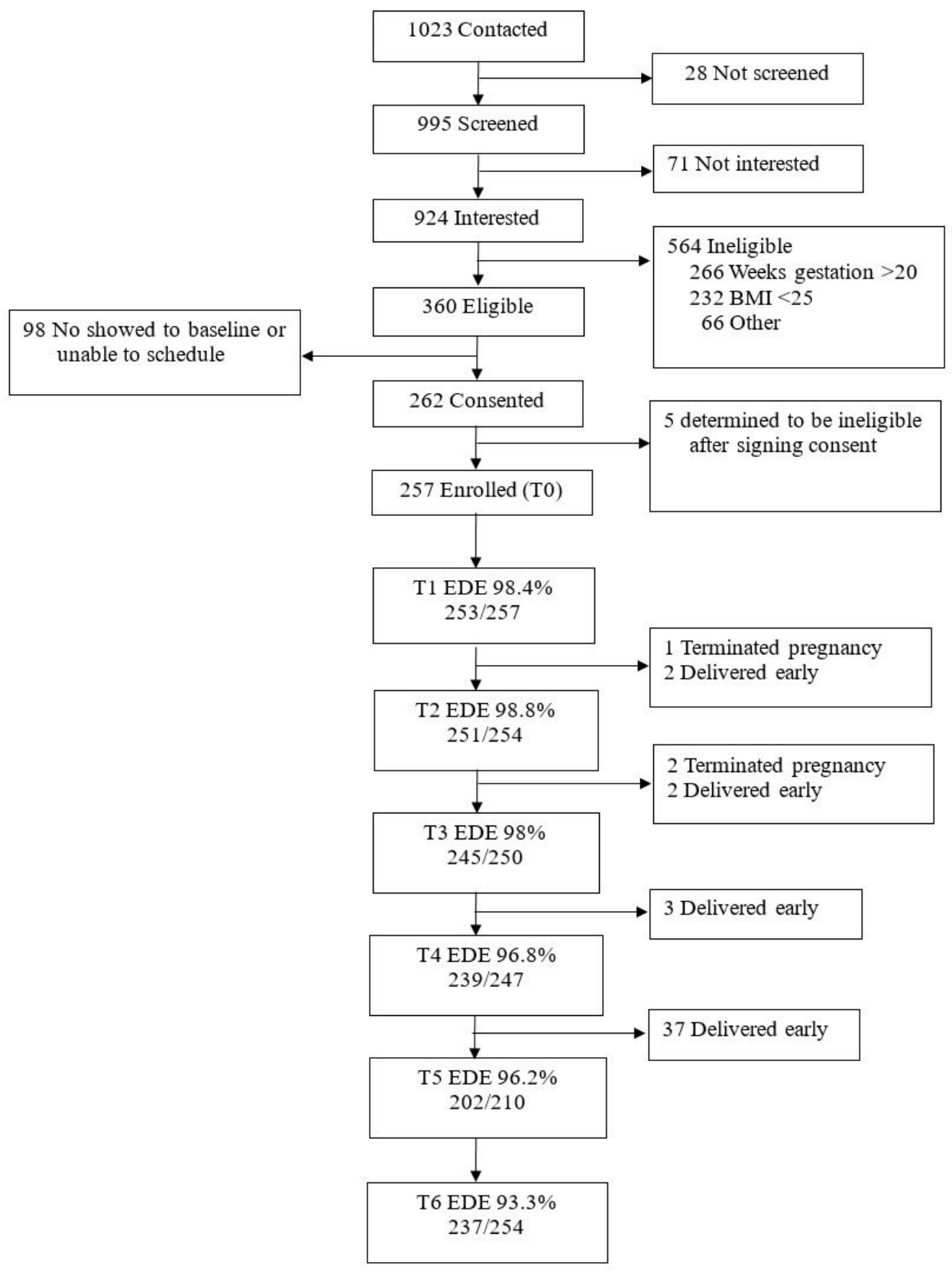

\section{Figure 1}

Participant Flow and EDE completion rates 